\title{
DE
}

DE GRUYTER

OPEN

BULGARIAN ACADEMY OF SCIENCES

CYBERNETICS AND INFORMATION TECHNOLOGIES • Volume 14, No 1

Sofia • 2014

Print ISSN: 1311-9702; Online ISSN: 1314-4081

DOI: 10.2478/cait-2014-0007

\section{Programming Learning Style Diagnosis Scheme Using PSO-Based Fuzzy Knowledge Fusion}

\author{
Jin Gou, Meizhen Chen, Wei Luo, Feng Hou \\ College of Computer Science and Technology, Huqiao University, Xiamen 361021, Fujian, China
}

\begin{abstract}
Different students have different learning styles, which are corresponding to their performances and make them behave differently in the learning process. Discovering the learning style of the students can help the development of teaching plans the students would accept more likely. It is a pity that few people dedicate to programming the learning style diagnosis. In view of the learning style, which is always closely linked with the learning performance, the programming learning behavior is introduced to programme the learning style diagnosis. This paper identifies the learning style of programming students in the learning process through their behavior preferences. To make the diagnosis more accurate, Particle Swarm Optimization (PSO) algorithm is introduced. The experiments invite junior students, senior students, graduate students and teachers of the College of Computer Science and Technology in the authors' university to fill out questionnaires as data. The experimental results show that PSO provides a great contribution.
\end{abstract}

Keywords: Learning style, programming learning behavior, particle swarm optimization, fuzzy knowledge fusion.

\section{Introduction}

People learn through various ways. Most have their own model to recognize or understand things and process stimuli or information. Learner's specific cognitive, 
emotional and physiological behavior constitute the learning style. A learning style can also be called a relatively stable learning mode. This style reflects the interaction with the environment. The unique style of learning influences the performance in the learning process. Researchers have proposed various learning style models according to their experiences and describing different viewpoints, such as Fleming's VAK/VARK model [1], Kolb's model [2] and FelderSilverman's model [3]. Finding out the learning styles of students and selecting the suitable teaching methods for them will greatly enhance the quality of teaching and learning. Many people are engaged in this [4, 5, 6]. This paper uses FelderSilverman's Learning Style Model (FSLSM). The details are discussed in the folowing sections.

FSLSM has been widely applied to computer industry by the scholars since it has been proposed [7, 8, 9]. But there is little information available in literature about programming learning styles diagnosis $[10,11]$. In the existing study of determining the programming learning styles, most scholars use the level as a measure of the provided indicators for the standard. This approach processes the diagnosis of the learning style more efficiently, but inadequacies also exist. Deficiencies will be discussed below. Programming learning behavior preferences are introduced as indicators by the authors. Furthermore, until recently, there is a lack of knowledge about trying to diagnose a complete model, than just draw one or some styles. This study is accomplished to determine the complete fourdimensional learning style model.

In this paper the relationship between the learning styles and diagnostic indicators is regarded as knowledge. It is certainly a kind of fuzzy knowledge. To optimize the knowledge, Particle Swarm Optimization (PSO) algorithm which learns from group information sharing, is introduced. The initial knowledge base is then ameliorated step by step through the training of sample sets. Finally it converges to the optimal value. In order to improve the efficiency of the algorithm, some changes are made to the coding which brings much convenience. To determine the efficiency of the optimization process, multiple test sets participated into the experiment.

The rest of the paper is organized as follows: Section 2 presents the theory and details of the learning style, and of the programming learning in Section 3. Section 4 describes the PSO algorithm and represents the fuzzy knowledge fusion. Experimental design and analysis are presented in Section 5. Finally, the paper is concluded in Section 6.

\section{Learning style}

The style of learning and teaching is one of the questions people have studied for a long time. It is clear that understanding the learning style of students can help a teacher to teach according to their aptitudes. The learning style reflects the performance of students in various aspects of the process of learning at different levels. The (FSLSM) used in this paper describes learning styles in four dimensions: Information Processing (IPr) (active and reflective), Information 
Perception (IPe) (sensing and intuitive), Information Reception (IR) (visual and verbal), and Information Understanding (IU) (sequential and global), which means that each student has four and only four categories.

\subsection{FSLSM}

FSLSM was processed in 1988, as a five-dimensional model with ten categories . It is then improved into the familiar four-dimensional and eight categories FSLSM [3]. Usually four dimensions are divided into processing, perception, reception and understanding, which can depict a complete learning style from different aspects.

\subsubsection{Information processing}

Information processing means perceiving information into knowledge. In this process the students can be divided into two categories: active and reflective (active experimentation and reflective observation) [12]. The active learners are involved in participation in discussions or applications, explanation to others, or test to hold information. In other words, they prefer the teamwork. Reflective learners are unable to process the information when they are not provided by any chance to think about the information presented. Reflective learners work better by themselves or with at most one other person.

\subsubsection{Information perception}

There are two ways people tend to perceive the word, sensing and intuition [13]. Sensors are involved in the process of observing, which means gathering data through senses. At the same time, intuitors percept indirectly through unconscious speculation, imagination, and intuition. Sensors are good at facts, not facing complex and unexpected situations. On the contrary, intuitions tend to find out the possibility and relationship between things, like innovation but not repeating. Sensors are very patient with details; they are more practical and careful than the intuitive ones. At the same time the intuitive people are good at grasping a new concept, work faster and are more innovative than the sensing type.

\subsubsection{Information reception}

FSLSM divides the way people receive information into two kinds: the first include visual sights, pictures, diagrams and symbols; the second include verbal-spoken and written words. Visual learners are good at remembering what they see, such as pictures, diagrams, flowcharts, image, video and presentation of content. Shown something but not told to, makes visual learners remember better. Differently, verbal learners are better in receiving information from the text and oral explanation. To a verbal learner, a word is much better than thousand pictures, whether spoken or written. 


\subsubsection{Information understanding}

In the part of knowledge understanding, the sequences solve problems by linear steps, keeping up with the previous step in every step. The global learners make an intuitive step and sometimes get solutions suddenly even if it is hard to explain how they work. Sequential learners tend to find answers step by step. Comprehensive learners may be able to solve complex problems more quickly or once they catch the main part and combine them in new ways. Sequential learners may be strong in convergent thinking and analysis, while global learners may be better at divergent thinking and synthesis.

\subsection{ILS}

Felder and Soloman propose the Index of Learning Styles (ILS), which is a 44items questionnaire for diagnosis of the learning style based on FSLSM [11, 13]. The questionnaire provides four values, between +11 and -11 , representing the learner's learning style preferences of each dimension. The four values are derived through the questionnaire to determine the learning styles of the four dimensions. For example, when someone gets a result like $+8,-9,+4,-5$, his learning styles may be $\{$ active, intuitive, visual, global $\}$. On the contrary, if the results are $-9,+8$, $-4,+5$, the styles will be \{reflective, sensing, verbal, sequence . Several scholars have studied and judged the reliability and validity of ILS since it was proposed. Fortunately, most of the results indicate the reliability and appropriateness of the ILS $[17,18]$.

In the experiments, the authors have used ILS to get the learning styles of students. Since the ILS has been proved to be reliable, the learning styles diagnosed by ILS are considered credible.

\section{Programming learning}

In order to provide some help to some of their fellow engineering professors at engineering education, Dr. Silverman and Dr. Fleder have proposed FSLSM according to their expertise and experience. FSLSM has indeed caused great repercussions and has been quoted by people from all aspects of life. It is applied to different areas of the field for it processed new proposals for teaching and learning. Some combine FSLSM with foreign and second languages [15]. Some use FSLSM to investigate the learning style preferences of a group of undergraduate pharmacy students [19]. Scholars also associate the learning style with English learning of native speakers and nonnative speakers online [20], face-to-face instruction learning [21].

As for computer professionals, many researchers have devoted to study FSLSM in the field of computer application and its effect on the learning process, etc. For example, a recommendation module of a Programmed tutoring system that can automatically adapt to the learners' interest and knowledge level is used to test the learner's learning style and learning habits [7], the relationship between learning 
styles and cognitive traits and its benefits in web-based educational systems [8], the automatic detection of learning styles for an e-learning system [9], etc.

Moreover, there are some researchers devoted to determine the programming learning styles by their behaviors in the learning process, most of the time twelve indicators are selected to measure their style $[10,11]$ called Programming Behaviors.

\subsection{Indicators}

To diagnose learning styles, some specific indicators are required. For a programming learning style, the researchers put forward twelve indicators: Cooperative Programming (C.P), Operation Application (O.A), Individual Programming (I.P), Method Design (M.D), Case Study (C.S), Theory Study (T.S), Diagram Illustration (D.I), Text Illustration (T.I), Sequential Order (S.O), Single Concept (S.C), Interlock Order (I.O), and Comprehensive Concept (C.C). The twelve represent e-Portfolio, programming learning performances [10, 11], etc. This paper names the indicators as Programming Learning Behaviors. Apparently, the twelve indicators can derive a complete FSLSM.

C.P, O.A, I.P, M.D can be used to distinguish between information processing. According to students' attitude toward C.P, O.A, whether a student is interested in processing information through hands, or enjoy team working can be found out. Through I.P and M.D, which detect students' interest in process information by thinking and the hobby of being alone, the reflective type will be determined. In other words, the four indicators above mentioned can introduce the active and reflective learners in programming learning. Sensors and intuitors can be detected by C.S and T.S. During a case study, the student may show he is surprisingly attentive to detail. Those are the students we call sensing learners. A theory study deal with the concept is definitively something intuitive learners are good at. The dimension Information Reception can be determined by D.I and T.I. D.I include diagrams, pictures, time lines, flow charts, demonstrations and films. It means that D.I exactly equals to what visual learners should be. In the same way, T.I can be used to detect verbal learners, since it contains word-written and spoken explanations. The last four indicators S.O, S.C, I.O and C.C detect Information Understanding. Sequential learners enjoy looking for a solution through a linear and logical way. The common ground between sequential learners and S.O, S.C can be found by this way. Due to the divergent thinking and synthesis, the global learners would prefer I.O and C.C than sequential learners. It is obvious that a student's learning style is not so single. You cannot draw a FSLSM by one or two indicators. The links between each index can also produce impacts on the last four-dimensional style. The twelve detect a FSLSM of each student.

\subsection{Programming learning behavior preferences}

Some people measure a level of twelve indicators and diagnose one or more learning styles successfully $[10,11]$. But as previously mentioned the authors believe that each student must have one of only four learning styles. This study is 
conducted to determine the Four-dimensional FSLSM. Furthermore, the four learning styles are corresponding to the four dimensions of the model one to one. FSLSM mentioned later will always be considered as a four-dimensional model containing four learning styles.

Another difference of this paper is that the measure of twelve indicators is not high and low, but a degree of preference. That means there is no longer "Amy's cooperative programming is high" or "Amy does quite good in cooperative programming" but "Amy really likes cooperative programming" or "Amy really hates cooperative programming". Since the learning style is more like a kind of abstract emotion rather than ability, the preferences are suitable for reflecting the learning styles. Known to all, preferences are emotions, "I like it" or "I hate it", and the level is more often regarded as ability "I'm good at this" or on the contrary.

In the process of obtaining sample data and rule sets, those who fill in the form are required to evaluate the indicators given within the range from -11 to +11 . +11 means he/she likes it very much and -11 represents the extreme dislike. Between these, there may be "not matter". A score is calculated based on the given membership function to determine their level of interest in the correponding project. Thus the linguistic values of the Programming Learning Behaviors would be :\{Dislike, Not Matter, Like $\}(\{D . N, L\})$.

\section{PSO-based Fuzzy Knowledge Fusion}

The operation of integrating multiple Fuzzy Knowledge Bases (FKBs) is Fuzzy Knowledge Fusion. Since the optimal fusion in this paper has the same purpose with text classification, both divide the sample into its category base unknown properties, the authors introduce recall and precision [22] to inspect the performance of PSO algorithm. The recall examines the ratio of correct classification under matching properties. The precision validates matching categories. The fitness function will be expressed as follows:

$$
\text { Fitness }(\text { FKB })=\frac{2 \times \text { Recall }(F K B) \times \text { Precision }(F K B)}{\text { Recall }(F K B)+\text { Precision }(F K B)} .
$$

PSO algorithm is a simulation of the flock social model based on the idea of swarm intelligence optimization [23]. The flock moves closer to the optimal solution through information sharing across the group. Kennedy and Eberhart proposed PSO in 1995 and developed it into a heuristic search technology. PSO algorithm flowchart is shown in Fig. 1.

One particle stands for one potential solution of the problem, a rule in this paper. The search results are finally measured by evaluating the function of fitness. Each particle has two characters: position and velocity. 
Particles tend to the optimal solution by adjusting their position, which depends on their own experiences and the surrounding neighbors. The velocity vector contains this information, driving the whole optimization process:

$$
V_{\mathrm{i}}^{t+1}=\omega V_{i}^{t}+C_{1} R_{1}\left(P_{i}^{t}-X_{i}^{t}\right)+C_{2} R_{2}\left(G_{i}^{t}-X_{i}^{t}\right),
$$

$$
X_{\mathrm{i}}^{t+1}=X_{i}^{t}+V_{i}^{t+1} \text {. }
$$

Here $X_{i}^{t}, V_{i}^{t}$ denote the position and velocity of the $i$-th particle in the $t$-th iteration; $\omega$, the inertia weight of the particle; $C_{1} R_{1}$ and $C_{2} R_{2}$ are called stochastic factors; $P_{i}^{t}$, the best solution of the $i$-th particle; and $G_{i}^{t}$, the best solution in the history.

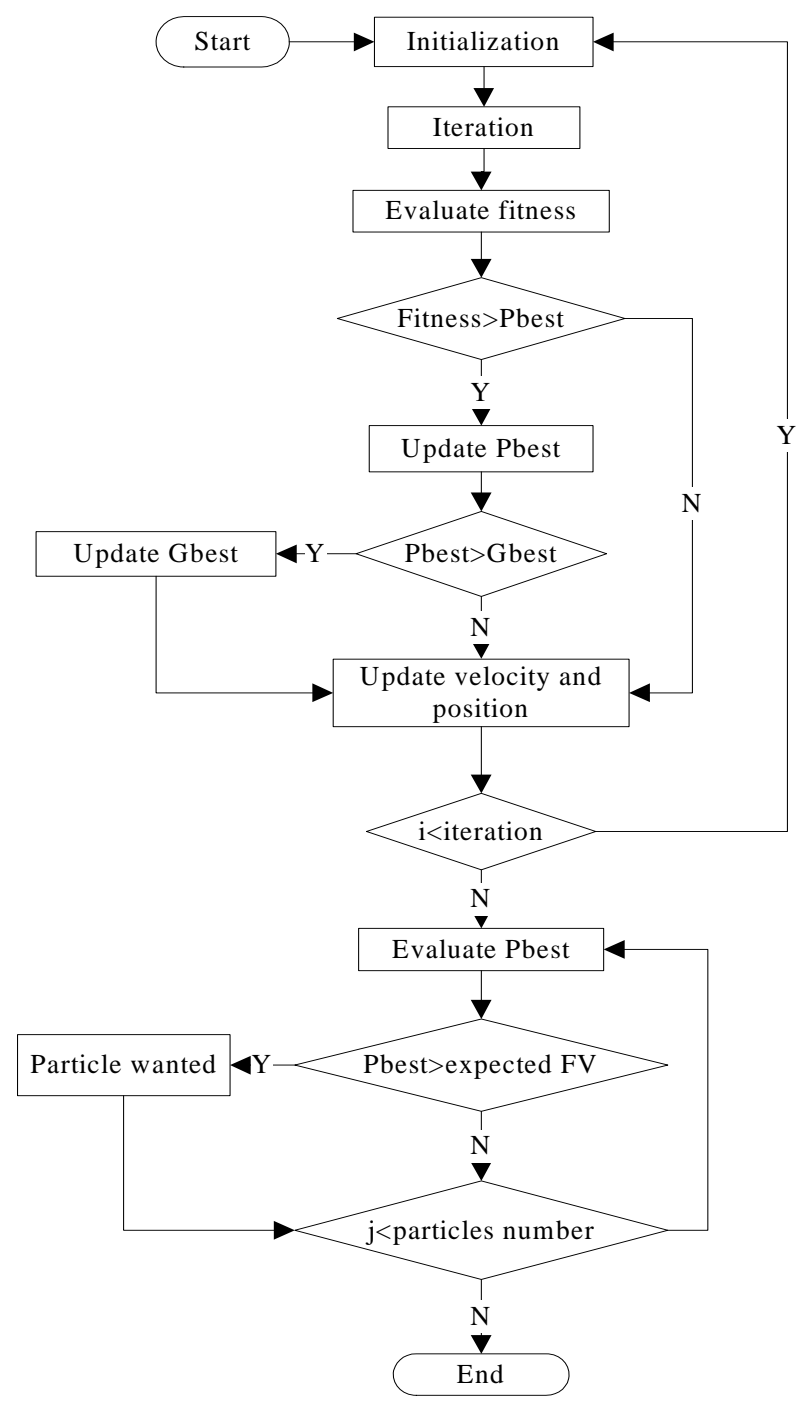

Fig. 1. PSO algorithm flowchart 
Table 1. PSO algorithm for FKBs integration

\begin{tabular}{|l|}
\hline Generate an initialize swarm of fuzzy knowledge base \\
Initialize Pbest, Gbest, velocities and positions \\
While scheduled fitness value or scheduled iteration is \\
not satisfied \\
For $i=1$ to FKB-population size \\
Calculate each particle's fitness value \\
If the fitness value is better than the Pbest in \\
history \\
Update Pbest by the current value \\
If the fitness value is better than the Gbest \\
Set the current value as the new Gbest \\
For $i=1$ to FKB-population size \\
Refresh particle velocity according to (2) \\
Refresh particle position according to (3)
\end{tabular}

\subsection{Fuzzy knowledge representations}

According to fuzzy knowledge theory, the linguistic value of the property is defined as: $\{$ Not matter, Like, Dislike $\}(\{\mathrm{N}, \mathrm{L}, \mathrm{D}\})$. In the paper, the trigonometric function is used to detect the membership of the related properties [24]. It is shown in Fig. 2.

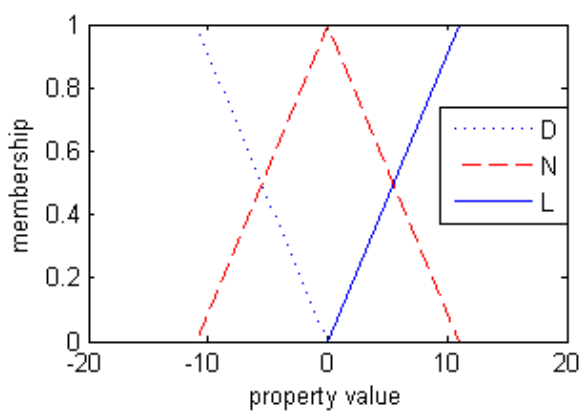

Fig. 2. The membership for the properties

Then the rules designed by the teachers will be as follows:

$$
\begin{aligned}
& \mathrm{C} . \mathrm{P}=\mathrm{L} \cap \mathrm{O} . \mathrm{A}=\mathrm{D} \cap \mathrm{I} . \mathrm{P}=\mathrm{N} \cap \mathrm{M} . \mathrm{D}=\mathrm{D} \cap \mathrm{C} . \mathrm{S}=\mathrm{N} ; \\
& \text { if } \cap \mathrm{T} . \mathrm{S}=\mathrm{D} \cap \mathrm{D} . \mathrm{I}=\mathrm{L} \cap \mathrm{T} . \mathrm{I}=\mathrm{D} \cap \mathrm{S} . \mathrm{O}=\mathrm{N} \cap \mathrm{S} . \mathrm{C}=\mathrm{L}, \\
& \bigcap \mathrm{I} . \mathrm{O}=\mathrm{DIC} . \mathrm{C}=\mathrm{N}, \\
& \text { then } \begin{array}{l}
\mathrm{IPr}=\text { activeIIPe=sensingIIR=visual } \\
\bigcap \mathrm{IU}=\text { global. }
\end{array}
\end{aligned}
$$

\subsection{Particle encoding}

In this paper the rules are encoded as particle and then PSO algorithm makes an iteration and moves them to the optimal position. Each particle represents one rule in the rule set. Some researchers make a Binary code, since it is easy to operate and handle, but the codes are also too long and sometimes reduce the efficiency of algorithm. The authors chose integer coding for a limitation and less of the 
linguistic value of the property and the type of result. A composed of $n$-th particles swarm will be expressed in Table 2 .

Table 2. Particle encoding

\begin{tabular}{|c|c|}
\hline$X_{1}$ & $\left(1,2, \ldots 2, \ldots, l_{1}\right)$ \\
\hline$X_{2}$ & $\left(0,2, \ldots 1, \ldots, l_{2}\right)$ \\
\hline$\ldots$ & $\ldots$ \\
\hline$X_{\mathrm{i}}$ & $\left(0,2, \ldots 1, \ldots, l_{i}\right)$ \\
\hline$\ldots$ & $\ldots$ \\
\hline$X_{n}$ & $\left(0,1, \ldots 2, \ldots, l_{n}\right)$ \\
\hline
\end{tabular}

Here $l_{i}$ represents the last dimension of $i$-th particle. Since a rule has twelve properties and four results, each particle is defined with a length of sixteen (Fig. 3).

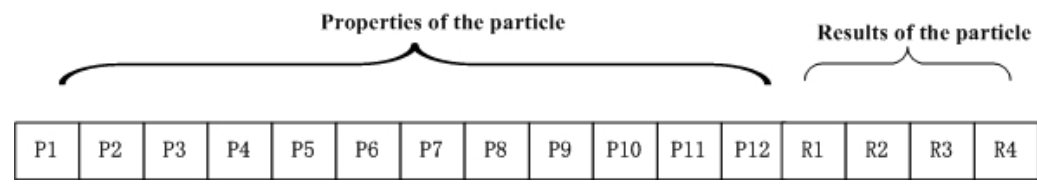

Fig. 3. The original image

A property of one particle is defined as a triple $\{\mathrm{N}, \mathrm{L}, \mathrm{D}\}$, and each linguistic value has two options: \{active/reflective, sensing/intuitive, visual/verbal, sequential/global $\}$. The authors use $\{0,1,2\}$ to represent $\{N, L, D\}$. If the result is of the first type, encode it as " 1 ", otherwise encode as " 2 ". In this way, the example rule:

C.P=LIO.A=DII.P=NIM.D=DIC.S=N;

if IT.S=DID.I=LIT.I $=$ DIS.O=NIS.C $=\mathrm{L}$

\section{กI.O=DIC.C $=\mathrm{N}$}

then

$\mathrm{IPr}=$ activeIIPe=sensingIIR=visual

กIU=global.

It is encoded as shown in Fig. 4.

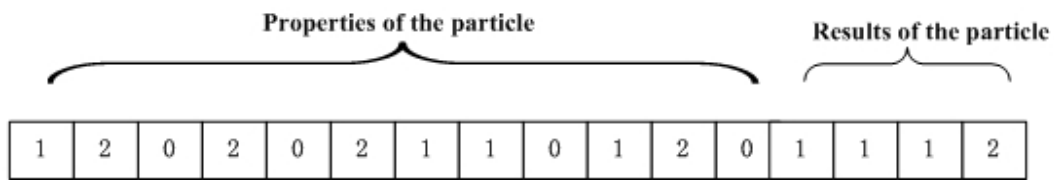

Fig. 4. Particle expression of the given example

\subsection{Fitness function}

The fitness determined by the Recall and Precision is an important indicator to measure the fusion effect. In view of the learning style, which has always four dimensions in this paper, the authors define the Recall and Precision functions as the following equations: 


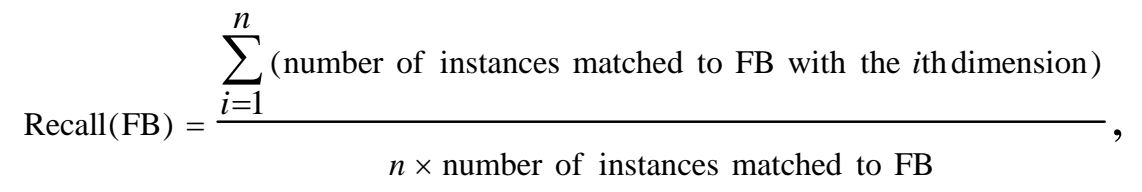

$$
\text { Precision }(\mathrm{FB})=\frac{\text { number of instances matched to FB }}{\text { number of instances matched to FB with results part }} \text {, }
$$

where $n$ denotes the dimension of the learning style, which equals to four in this paper.

\subsection{Overflow handling}

To prevent the particles from being out of range in the process of updating, some measures are usually taken. Methods available now are absorbing wall, reflecting wall, cyclic wall, etc. This paper chooses a cyclic wall which cycles the transponder particles back to search space when the particles flying out of the any dimension boundary [25]. The particles position adjustment formula are:

$$
\begin{gathered}
X_{i j}=\left\{\begin{array}{cc}
(-1) \times X_{i j} \bmod \left(l_{i}+1\right) & X_{i j}<0, \\
X_{i j} & X_{i j}=0, \\
X_{i j} \bmod \left(l_{i}+1\right) & X_{\mathrm{ij}}>0 ;
\end{array}\right. \\
X_{i j}=\left\{\begin{array}{cc}
(-1) \times X_{i j} \bmod \left(l_{i}\right)+1 & X_{i j}<0, \\
X_{i j} \bmod \left(l_{i}\right)+1 & X_{i j}>0 .
\end{array}\right.
\end{gathered}
$$

Equation (6) is used for updating bits representing properties and programming learning behaviors in a particle. Equation (7) is used for output, which means the learning style types. In these equations, $X_{i j}$ denotes the $j$-th bit of $i$-th particle, and $l_{i}$ is the maximize integer used for representing the $i$-th particle.

\section{Experiments}

In order to verify that PSO can help programming learning preferences-based learning styles diagnosis, the fitness depending on recall and precision, is used for comparison. The experiments invite seniors, juniors, graduates and professional teachers to fill out the corresponding questionnaires. The authors obtain the sample data, the test data and rules from the questionnaires. Then PSO is joined in to optimize the data. Since there is some uncertainty in PSO caused by its randomness, each experiment is carried out 20 times. The average values of fitness, recall and precision are shown as figures. In each iterative process some values of fitness, recall and precision are also listed in the Table 3. 
Table 3. Experimental procedure

\begin{tabular}{|l|}
\hline $\begin{array}{l}\text { Step 1. Distribute questionnaires among junior, senior and } \\
\text { graduate students to obtain data (ILS and } \\
\text { programming behavior preferences) }\end{array}$ \\
Step 2. Determine each student's learning style by ILS \\
Step 3. Get the rules proposed by teachers \\
Step 4. Swarm intelligence algorithm optimization rules \\
Step 5. Determine the fitness
\end{tabular}

\subsection{Sample data acquisition}

In order to get the students' learning styles and learning preferences, a questionnaire is distributed to juniors, seniors and graduate students in the experiment. The questionnaires contain ILS and twelve more questions for determining the programming learning preferences. Through ILS, the four-dimensional learning styles of a student can be determined. Then the correspondence selects between the programming learning preferences and the learning styles of each student.

The experiment includes 100 junior students, 100 senior students and 20 graduate students to complete the questionnaires. The four-dimensional learning style model of each student is identified by the first 44 questions. The followed 12 answers are converted to the linguistic value by the membership function shown in Fig. 2. Then the data obtained from the questionnaire is expressed as given in Table 4. In Table 4, A represents Active, R - Reflective, I - Intuitive, S - Sensing, Vi Visual, Ve - Verbal, S - Sequential and G represents Global.

The 220 data is divided into three training sets and one test sample set as shown in Fig. 5. In addition, hybrid here covers 20 cases of seniors, 20 cases of juniors and 20 cases of graduates.

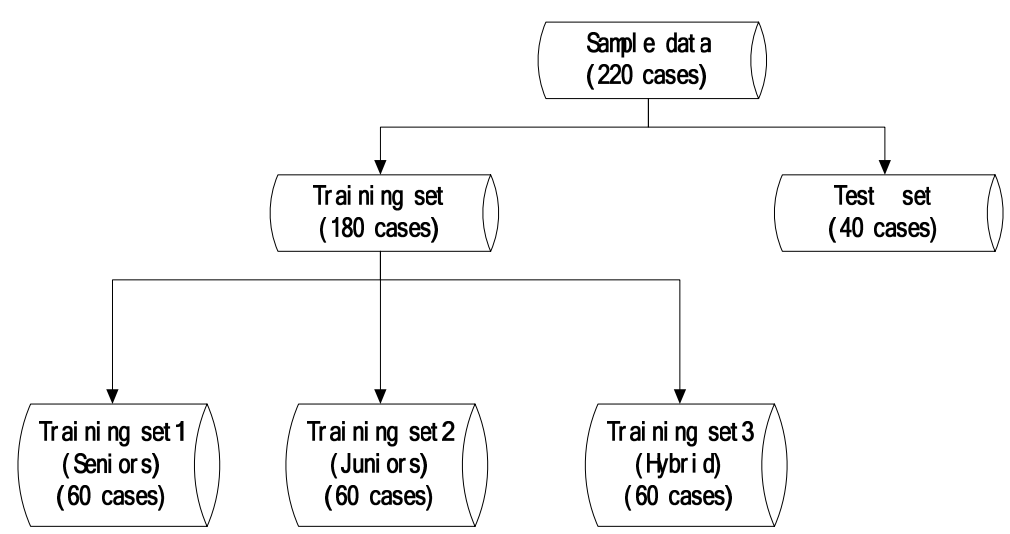

Fig. 5. Data partitioning 
Table 4. Examples of sample data

\begin{tabular}{|l|l|l|l|l|l|l|l|l|l|l|l|l|l|l|l|}
\hline C.P & I.P & O.A & M.D & C.S & T.S & D.I & T.I & S.O & I.O & S.C & C.C & Pr & Pe & R & U \\
\hline L & N & N & D & N & D & N & N & N & N & N & N & A & I & Vi & S \\
\hline N & N & L & D & N & L & L & N & L & N & N & N & R & I & Vi & G \\
\hline D & N & N & L & D & N & D & N & L & N & N & L & R & I & Vi & S \\
\hline D & D & D & D & D & D & L & N & L & N & N & L & A & S & Vi & G \\
\hline N & N & N & D & D & N & D & L & N & L & N & L & A & S & Vi & S \\
\hline L & N & N & N & L & N & L & N & N & N & N & L & R & I & Ve & G \\
\hline N & D & L & N & L & L & L & L & L & D & D & L & A & I & Vi & G \\
\hline L & D & N & D & L & L & L & N & N & D & N & N & R & I & Vi & S \\
\hline L & N & N & D & L & D & L & L & N & L & N & N & A & S & Ve & S \\
\hline D & D & N & D & D & D & D & D & D & D & N & L & R & S & Vi & G \\
\hline
\end{tabular}

\subsection{Experts proposed rules}

There are several different methods to obtain rules, such as extracting rules by a genetic algorithm, accessing rules through specific tools, etc. The experts proposed rules are adopted in the experiments. Since a professional teacher can be regarded as an educational expert in this field, 15 teachers are invited to put forward the rules believed to be reasonable, based on their experience in teaching at the College of Computer Science and Technology of the authors' university. Each teacher is requested to propose from 7 up to 15 reliable rules. One of the rule sets proposed by the teacher is also given in Table 5. In Table 5 A represents Active, $\mathrm{R}$ - Reflective, I - Intuitive, S - Sensing, Vi - Visual, Ve - Verbal, S - Sequential and G represents Global. The fitness, recall and precision of the twelve initial rule sets are shown in Table 6.

Table 5. Examples of sample data

\begin{tabular}{|l|l|l|l|l|l|l|l|l|l|l|l|l|l|l|l|}
\hline C.P & I.P & O.A & M.D & C.S & T.S & D.I & T.I & S.O & I.O & S.C & C.C & Pr & Pe & R & U \\
\hline L & N & N & D & N & D & N & N & N & N & N & N & A & I & Vi & S \\
\hline N & N & L & D & N & L & L & N & L & N & N & N & R & I & Vi & G \\
\hline D & N & N & L & D & N & D & N & L & N & N & L & R & I & Vi & S \\
\hline D & D & D & D & D & D & L & N & L & N & N & L & A & S & Vi & G \\
\hline N & N & N & D & D & N & D & L & N & L & N & L & A & S & Vi & S \\
\hline L & N & N & N & L & N & L & N & N & N & N & L & R & I & Ve & G \\
\hline N & D & L & N & L & L & L & L & L & D & D & L & A & I & Vi & G \\
\hline L & D & N & D & L & L & L & N & N & D & N & N & R & I & Vi & S \\
\hline L & N & N & D & L & D & L & L & N & L & N & N & A & S & Ve & S \\
\hline D & D & N & D & D & D & D & D & D & D & N & L & R & S & Vi & G \\
\hline
\end{tabular}


Table 6. The performance of the 12 initial rule bases

\begin{tabular}{|l|l|l|l|}
\hline Rule bases & Fitness & Recall & Precision \\
\hline Base1 & 0.4298 & 0.3214 & 0.7937 \\
\hline Base2 & 0.46337 & 0.3500 & 0.8125 \\
\hline Base3 & 0.2562 & 0.2000 & 0.4143 \\
\hline Base3 & 0.2562 & 0.2000 & 0.4143 \\
\hline Base4 & 0.4200 & 0.3935 & 0.4923 \\
\hline Base5 & 0.3453 & 0.2375 & 0.7250 \\
\hline Base6 & 0.3488 & 0.2429 & 0.7135 \\
\hline Base7 & 0.4630 & 0.3463 & 0.8265 \\
\hline Base8 & 0.6318 & 0.5500 & 0.8288 \\
\hline Base9 & 0.4473 & 0.3531 & 0.7576 \\
\hline Base10 & 0.2766 & 0.2000 & 0.5188 \\
\hline Base11 & 0.2483 & 0.2062 & 0.3795 \\
\hline Base12 & 0.3754 & 0.2821 & 0.6765 \\
\hline Base13 & 0.4088 & 0.3164 & 0.67474 \\
\hline Base14 & 0.2795 & 0.2000 & 0.5330 \\
\hline Base15 & 0.2903 & 0.2000 & 0.5733 \\
\hline
\end{tabular}

\subsection{Process of optimization}

PSO algorithm is introduced to improve the accuracy of diagnosis. Samples and rules are obtained through the questionnaire. The experiment then steps into knowledge integration, optimizing the knowledge through integration of multiple rule bases. The sample data is divided into a training sample set and a testing sample set. An optimized rule base will be found after trained by the training set. The optimal fusion is to maximize the recall and keep precision.

\subsection{Results and analysis}

The experimental results show that in the process of training or iterative process, the recall and fitness rise steadily until they reach a relatively high value, meanwhile the precision produces a smaller decrease. Since there is some uncertainty in PSO, the experiment loops for twenty times. During the iteration of training, the average performance is shown in Figs 6 and 7. The iteration in the 9-th cycle is also given in Table 7. Table 8 shows the performance of the rule base on a test set during the iteration in the 12th cycle.

The results obtained through the training process show that PSO algorithm would train the initial rules to improve the fitness, and recall and keep precision. To verify the accuracy, the test set is introduced in each iteration.

Compare the values of fitness, precision and recall of the 9th cycle of training in Table 7 with the initial values in Table 4, it can be concluded that the training set makes the fitness and recall steady increasing and keeps precision in every iterative process. Furthermore, the value of the 12th cycle of testing in Table 8 verifies the efficiency of the experimental results. This means that the experiment not only runs well on training sets, but also on testing sets. 
Table 7. The performance of rule base on training set during the iteration in the 9th cycle

\begin{tabular}{|l|l|l|l|l|}
\hline Training set & Iteration & Fitness & Recall & Precision \\
\hline \multirow{5}{*}{ Hybrid } & 1 & 0.5746 & 0.4754 & 0.8428 \\
\cline { 2 - 5 } & 100 & 0.751 & 0.6833 & 0.8531 \\
\cline { 2 - 5 } & 200 & 0.7768 & 0.7371 & 0.8405 \\
\cline { 2 - 5 } & 300 & 0.7732 & 0.7312 & 0.8449 \\
\cline { 2 - 5 } & 400 & 0.7687 & 0.726 & 0.8479 \\
\cline { 2 - 5 } & 500 & 0.7723 & 0.7267 & 0.8562 \\
\hline \multirow{5}{*}{ Seniors } & 1 & 0.5849 & 0.5003 & 0.8477 \\
\cline { 2 - 5 } & 100 & 0.7472 & 0.6903 & 0.8392 \\
\cline { 2 - 5 } & 200 & 0.7709 & 0.7382 & 0.8216 \\
\cline { 2 - 5 } & 300 & 0.7942 & 0.7866 & 0.8158 \\
\cline { 2 - 5 } & 400 & 0.8012 & 0.7944 & 0.8293 \\
\cline { 2 - 5 } & 500 & 0.7906 & 0.7793 & 0.8262 \\
\hline \multirow{5}{*}{ Juniors } & 1 & 0.6103 & 0.5057 & 0.8505 \\
\cline { 2 - 5 } & 100 & 0.7434 & 0.6869 & 0.8537 \\
\cline { 2 - 5 } & 200 & 0.8461 & 0.8977 & 0.8177 \\
\cline { 2 - 5 } & 300 & 0.8616 & 0.9294 & 0.8137 \\
\cline { 2 - 5 } & 400 & 0.8892 & 0.978 & 0.8175 \\
\cline { 2 - 5 } & 500 & 0.8856 & 0.9648 & 0.8271 \\
\hline
\end{tabular}

Observing Figs 6 and 7, it can be found from the two experiments that the training with the training set rule base can indeed improve the recall and maintain precision to a relatively stable value. The accuracy of the rule base continuously improves with the growth of iterations. Unfortunately, the precision can only be maintained at a relatively high and stable value. On the other hand, the hybrid containing three types of students can achieve a higher recall rate in the case of a relatively high precision. The reason may be that the training set contains more types of students.

Table 8 . The performance of the rule base on a test set during the iteration in the 12th cycle

\begin{tabular}{|l|l|l|l|l|}
\hline Test set & Iteration & Fitness & Recall & Precision \\
\hline \multirow{5}{*}{ Hybrid } & 1 & 0.5557 & 0.4549 & 0.8384 \\
\cline { 2 - 5 } & 100 & 0.7798 & 0.74 & 0.8497 \\
\cline { 2 - 5 } & 200 & 0.8507 & 0.8846 & 0.8345 \\
\cline { 2 - 5 } & 300 & 0.866 & 0.915 & 0.8355 \\
\cline { 2 - 5 } & 400 & 0.8716 & 0.9235 & 0.8398 \\
\cline { 2 - 5 } & 500 & 0.8705 & 0.9247 & 0.8358 \\
\hline \multirow{5}{*}{ Seniors } & 1 & 0.621 & 0.5353 & 0.8328 \\
\cline { 2 - 5 } & 100 & 0.8053 & 0.7963 & 0.8248 \\
\cline { 2 - 5 } & 200 & 0.8194 & 0.842 & 0.811 \\
\cline { 2 - 5 } & 300 & 0.8412 & 0.8806 & 0.8167 \\
\cline { 2 - 5 } & 400 & 0.8433 & 0.8872 & 0.8139 \\
\cline { 2 - 5 } & 500 & 0.8572 & 0.911 & 0.8168 \\
\cline { 2 - 5 } & 1 & 0.621 & 0.5258 & 0.853 \\
\cline { 2 - 5 } & 100 & 0.8053 & 0.8576 & 0.8386 \\
\cline { 2 - 5 } & 200 & 0.8194 & 0.9154 & 0.8293 \\
\cline { 2 - 5 } & 300 & 0.8412 & 0.9575 & 0.8302 \\
\cline { 2 - 5 } & 400 & 0.8433 & 0.9544 & 0.8323 \\
\hline \multirow{5}{*}{ Juniors } & 0.8572 & 0.9538 & 0.8326 \\
\hline
\end{tabular}




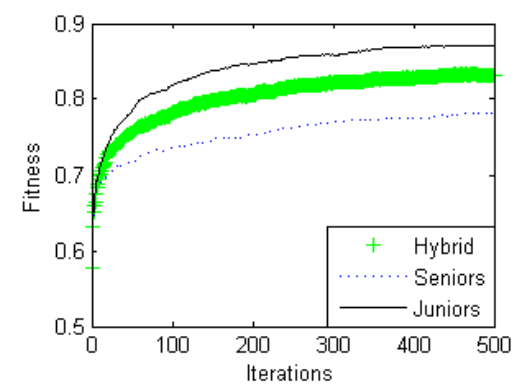

(a) Fitness

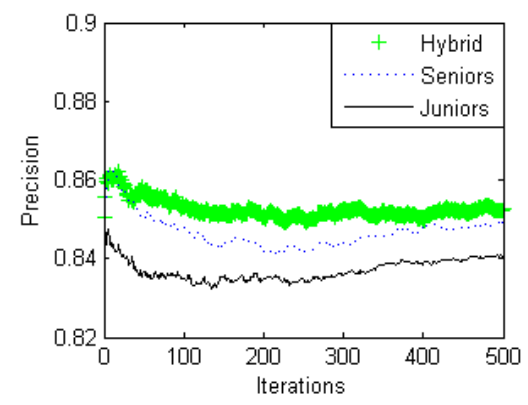

(b) Precision

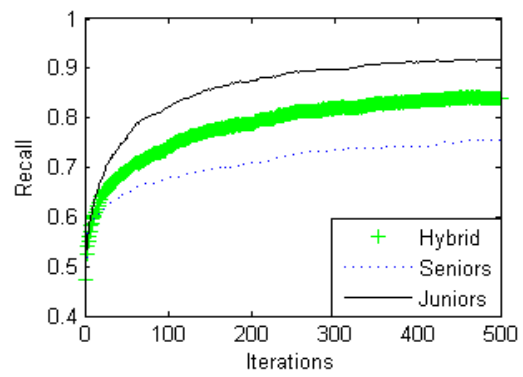

(c) Recall

Fig. 6. The average performance of the rule base on a training set during the iteration

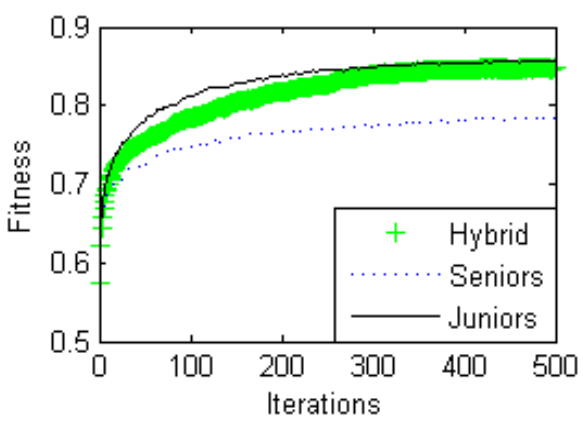

(a) Fitness

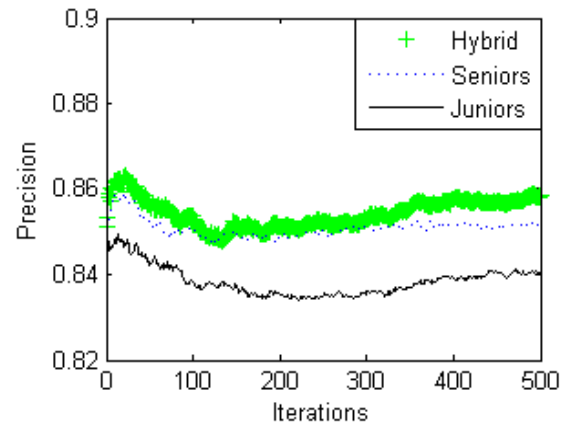

(b) Precision

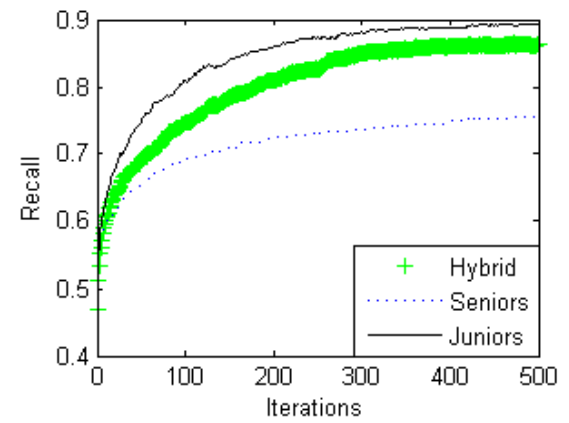

(c) Recall

Fig. 7. The average performance of the rule base on a test set during the iteration 


\section{Conclution}

This paper presents a programming learning behavior preferences-based learning style diagnosis model. The judgement of the students by a complete fourdimensional FSLSM is an innovation of the paper. PSO is used to cluster and optimize the original knowledge base. The experimental results shows that the fuzzy knowledge fusion processes the diagnosis more efficiently.

Acknowledgements: This work was supported by the National Natural Science Foundation of China (No 61103170) and the Program for Prominent Young Talents in Fujian Province University (No JA12005).

\section{References}

1. M a r c y, V. Adult Learning Styles: How the VARK Learning Styles Inventory Can be Used to Improve Student Learning. - Perspectives on Physician Assistant Education, Vol. 12, 2001, No 2, 117-120.

2. Kolb, D. A., R. E. B o y a tz i s, C. Ma i n e melis. Experiential Learning Theory: Previous Research and New Directions. - Perspectives on Thinking, Vol. 1, 2001, 227-247.

3. F e ld e r, R. M., L. K. S i lve r m a n. Learning and Teaching Styles in Engineering Education. Engineering Educatio, Vol. 78, 1988, No 7, 674-681.

4. M o r e, A. J. Native Indian Learning Styles: A Review for Researchers and Teachers. - Journal of American Indian Education,Vol. 27, 1987, No 1, 17-29.

5. F e l d e r, R. M. How Students Learn: Adapting Teaching Styles to Learning Styles. - In: Frontiers in Education Conf. on IEEE, 1988, 489-410.

6. Smith, L. H., J. S. Renzulli. Learning Style Preferences: A Practical Approach for Classroom Teachers. - Theory into Practice, Vol. 23, 1984, No 1, 45-50.

7. V e s i n, A. B., Et al. E-Learning Personalization Based on Hybrid Recommendation Strategy and Learning Style Identification. - Computers and Education, Vol. 56, 2011, No 3, 885-899.

8. Gra f, S., T. C. L i u, N. S. C h e n, S. J. Y a n g. Learning Styles and Cognitive Traits-Their Relationship and its Benefits in Web-Based Educational Systems. - Computers in Human Behavior, Vol. 25, 2009, No 6, 1280-1289.

9. Graf, S., T. Lin. The Relationship between Learning Styles and Cognitive Traits-Getting Additional Information for Improving Student Modeling. - Computers in Human Behavior, Vol. 24, 2008, No 2, 122-137.

10. H u a n g, A. F., S. J. Y a n g, M. W a n g, J. J. T s a i. Improving Fuzzy Knowledge Integration with Particle Swarm Optimization. - Expert Systems with Applications,Vol. 37, 2010, No 12, 8770-8783.

11. Huang, A. F., J. T. Wu, S. J. Y ang, W. Y. Hwang. The Success of Portfolio-Based Programming Learning Style Diagnosis: Exploring the Role of a Heuristic Fuzzy Knowledge Fusion. - Expert Systems with Applications, Vol. 39, 2012, No 10, 8698-8706.

12. He n d e rs o n, J. L. CG Jung's Psychology: Additions and Extensions. - Journal of Analytical Psychology, Vol. 36, 1991, No 4, 429-442.

13. Kolb, A. Y., D. A. Kolb., Learning Styles and Learning Spaces: Enhancing Experiential Learning in Higher Education. - Academy of Management Learning \& Education, Vol. 4, 2005, No 2, 193-212.

14. Felder, R. M., J. S p u rlin. Applications, Reliability and Validity of the Index of Learning Styles. - International Journal of Engineering Education, Vol. 21, 2005, No 1, 103-112.

15. Felder, R. M., E. R. Henriques. Learning and Teaching Styles in Foreign and Second Language Education. - Foreign Language Annals, Vol. 28, 1995, No 1, 21-31.

16. L it z i n g e r, T. A., S. H. L e e, J. C. W i s e, R. M. F e l d e r. A Psychometric Study of the Index of Learning Styles. - Journal of Engineering Education, Vol. 96, 2007, No 4, 309-319. 
17. V a n Z w a n e n b e r g, N., L. W il k i n s o n, A. A n d e r s o n. Felder and Silverman's Index of Learning Styles and Honey and Munford's Learning Styles Questionnaire: How Do They Compare and Do They Predict Academic Performance?. - Educational Psychology, Vol. 20, 2000, No 3, 365-380.

18. Gra f, S., T. C. Li u. Analysis of Learners' Navigational Behavior and Their Learning Styles in an Online Course. - Journal of Computer Assisted Learning, Vol. 26, , 2010 No 2, 116-131.

19. Willi a m s, B., T. Brown, J. E the ringt on. Learning Style Preferences of Undergraduate Pharmacy Students. - Currents in Pharmacy Teaching and Learning, Vol. 5, 2013, No 2, 110-119.

20. R e i d, J. M. The Learning Style Preferences of ESL Students. - TESOL quarterly, Vol. 21, 1987, No 1, 87-111.

21. N e u h a u s e r, C. Learning Style and Effectiveness of Online and Face-to-Face Instruction. - The American Journal of Distance Education, Vol. 16, 2002, No 2, 99-113.

22. Yang, Y. An Evaluation of Statistical Approaches to Text Categorization. - Information Retrieval, Vol. 1, 1999, No 1-2, 69-90.

23. K e n n e dy, J., R. E b e r h a rt. Particle Swarm Optimization. - In: IEEE International of First Conference on Neural Networks, 1995.

24. Z hou, X. F., L. J. Jiang, Z. L. S ong. A Classification System Based on Fuzzy Class Association Rules. - Journal of Computer Research And Development, Vol. 40, 2003, No 5, 651-656.

25. Che n, X., Q. Gu, Z. Y. W a n g, D. X. Che n. Framework of Particle Swarm Optimization Based Pairwise Testing. - Ruanjian Xuebao/Journal of Software, Vol. 22, 2011, No 12, 2879-2893 (in Chinese). 